\title{
Characterization of a spheromak plasma gun: The effect of refractory electrode coatings
}

\author{
M. R. Brown, a) A. D. Bailey III, ${ }^{\text {,) }}$ and P. M. Bellan \\ California Institute of Technology, Pasadena, California 91125
}

(Received 27 September 1990; accepted for publication 23 January 1991)

\begin{abstract}
In order to investigate the proposition that high- $Z$ impurities are responsible for the anomalously short lifetime of the Caltech spheromak, the center electrode of the spheromak plasma gun has been coated with a variety of metals (bare steel, copper, nickel, chromium, rhodium, and tungsten $)$. Visible light $(230-890 \mathrm{~nm}$ ) emitted directly from the plasma in the gun breech was monitored for each of the coated electrodes. Plasma density and temperature and spheromak lifetime were compared for each electrode. Results indicate little difference in gun performance or macroscopic plasma parameters. The chromium and tungsten electrodes performed marginally better in that a previously reported helicity injection effect [ Phys. Rev. Lett. 64, 2144 (1990) ] is only observed in discharges using these electrode coatings. There are subtle differences in the detailed line emission spectra from the different electrodes, but the spectra are remarkably similar. The fact that (1) contrary to expectations, attempts to reduce high- $Z$ impurities had only marginal effect on the spheromak lifetime coupled with (2) an estimate of $Z_{\text {eff }}<2$ based on a $0-D$ model suggests that it is not impurities but some other mechanism that limits the lifetime of small, cold spheromaks. We will discuss the general characteristics of the spheromak gun as well as effects due to the coatings.
\end{abstract}

\section{INTRODUCTION}

An important consideration for plasma configurations generated from high current density electrodes (e.g., spheromaks, ${ }^{1}$ de helicity injection discharges, ${ }^{2}$ Marshall gun, ${ }^{3}$ and Mather focus ${ }^{4}$ plasmas) is contamination from impurities. At high current densities, high- $Z$ electrode material (as well as low- $Z$ adsorbed impurity gases) can be sputtered or evolved into the discharge thereby raising $Z_{\text {eff }}$ and increasing the resistivity and radiated power from the plasma. The interaction of hot plasma with material limiters in large tokamaks and the concomitant increase in $Z_{\text {eff }}$ is a related issue. $^{5}$ Impurities have been a particular concern of spheromak researchers. ${ }^{6-9}$ The Caltech Spheromak Injection experiment $^{10,11}$ may be susceptible to this problem since high discharge currents $(<150 \mathrm{kA})$ are generated from small electrodes $\left(\sim 100 \mathrm{~cm}^{2}\right)$. We have noted that (1) our spheromak magnetic lifetimes $\left(\tau_{\text {life }}\right)$ are anomalously short compared with predictions of the resistive decay time and that (2) copious impurity line emission is observed throughout the discharge lifetime. In addition, we have observed ablation of the center electrode and sputtering of electrode material onto quartz vacuum fixtures. It has remained unclear whether there was a correlation between impurity content (i.e., $Z_{\text {eff }}$ ) and short $\tau_{\text {life }}$.

Spheromaks are force-free magnetofluid configurations with comparable toroidal and poloidal magnetic fluxes ${ }^{12}$ which are generated largely by currents flowing in the plasma itself. In force-free systems, internal $\mathbf{J} \times \mathbf{B}$ forces nearly balance so that the magnetic field structure of spheromaks is approximately governed by the eigenvalue equation

\footnotetext{
a) United States Department of Energy Fusion Energy Postdoctoral Research Fellow.

b) United States Department of Energy Fusion Science Research Fellow.
}

$\boldsymbol{\nabla} \times \mathbf{B}=\lambda \mathbf{B}$ (with $\lambda=$ constant). This equation must be solved subject to boundary conditions; if a perfectly conducting wall is assumed then image currents must flow to provide equilibrium fields. Because of their high plasma density and magnetic helicity content compared with tokamaks (the helicity $K=\int A \cdot B d^{3} x$ is relatcd to volume integrated field aligned current), spheromaks have been proposed as both a tokamak refueling and current drive scheme. ${ }^{13-15}$ The Caltech Spheromak Injection experiment has demonstrated both tokamak refueling and refluxing (current drive) by spheromak injection. ${ }^{10}$

Early spheromak researchers determined that impurity radiation (dependent on $Z_{\text {eff }}$ ) was the most important energy loss mechanism in their discharges. ${ }^{16}$ From bolometery measurcments it appeared that all the plasma magnetic energy was radiated by impurities during decay. In the larger, warm, nonradiation dominated CTX spheromaks, Barnes, et al. ascertained that enhanced particle $\operatorname{loss}^{6}$ and particle replacement power ${ }^{7}$ were the primary loss mechanisms. Particle replacement power is that required to ionize neutral gas and heat cold electrons to the bulk temperature in a particle confinement time. More recently, it has been recognized that an important loss mechanism in spheromaks is magnetic helicity dissipation due to electric fields in the plasma edge. ${ }^{8}$ The CTX group has inferred large edge electric fields due to the spheromak relaxation process. Since helicity dissipation depends on the electric field, helicity dissipation is greater at the edge in their discharges.

To understand the anomalous lifetimes in the Caltech Spheromak Injection Experiment and ascertain whether the cause is large impurity content, we embarked on a program of electrode coating and spheromak cleanup. In Sec. II, theoretical issucs concerning spheromak lifetimes, impurity production rates, $Z_{\text {eff }}$ and other mechanisms affecting $\tau_{\text {life }}$ are 
discussed. In Sec. III, the Caltech spheromak gun and the adverse role of impurities are described. In Sec. IV, our main spectroscopic results from the various coated electrodes are presented. In Sec. V, we close with a discussion of our results in the context of other experiments and a summary. Gun performance and operation are characterized in the appendices. Electromagnetic properties of the gun are discussed in Appendix A and we characterize the role of neutral gas in our experiment in Appendix B.

\section{THEORETICAL ISSUES}

\section{A. Spheromak lifetimes}

The spheromak resistive lifetime can be viewed as an $L / R$ decay time where $L$ is the spheromak inductance determined by the dimensions of the current path in the discharge and $R$ is the Spitzer resistance of the spheromak plasma. Turner et al. ${ }^{16}$ have pointed out that the magnetic diffusion equation governing spheromak resistive decay can be written:

$$
\frac{\partial B}{\partial t}=\frac{-\eta \lambda^{2} B}{\mu_{0}}
$$

where $\eta$ is the Spitzer resistivity ( $\eta \propto Z_{\text {eff }} T^{-3 / 2}$ ) and $\lambda$ is the eigenvalue in the equation for the force-free state $(\nabla \times \mathbf{B}=\lambda \mathbf{B})$. The $e$-folding time for the spheromak magnetic fields is then

$$
\tau_{\text {mag }}=\mu_{0} /\left(\eta \lambda^{2}\right) \text {. }
$$

We have noted that the magnetic decay time of our spheromaks is anomalously short compared to this simple Spitzer lifetime calculation. We have performed extensive magnetic measurements of the spheromak equilibrium injected into the empty Encore tokamak vessel ${ }^{17}$ and have determined from a fit of the data to a simple equilibrium model that $\lambda \cong 22 \mathrm{~m}^{-1}$. For a value of $T_{e}=7 \mathrm{eV}$ (measured with a double Langmuir probe) and assuming $Z_{\text {eff }}=1$ we calculate $\tau_{\text {mag }} \cong 100 \mu \mathrm{s}$ while the measured $e$-folding time $\tau_{\text {mag }} \cong 10 \mu \mathrm{s}$ in our spheromak (the total $\tau_{\text {life }} \cong 20 \mu \mathrm{s}$ ). Spheromaks which are not fully relaxed, and thus have smaller dimensions, have been measured with values of $\lambda$ up to $30 \mathrm{~m}^{-1}$ and fully relaxed spheromaks late in the decay phase of the discharge have $T_{e}$ as low as $4 \mathrm{eV}$. In these extreme cases, we calculate $\tau_{\text {mag }} \cong 50 \mu \mathrm{s}$. We conclude that there is an anomaly factor of 5-10 between the predicted and measured $e$-folding times of the magnetic fields of our spheromak discharges. Other researchers have noted a discrepancy between measured spheromak lifetimes and those predicted by simple Spitzer resistivity. Barnes et al. ${ }^{6}$ have reported a "resistance anomaly" of $1.6-4.0$ coupled with a $Z_{\text {eff }}$ of $1.3-$ 2.

It is important for spheromak injection experiments that the spheromak can traverse the distance from its source (a coaxial magnetized plasma gun in our case) to the center of the tokamak before the spheromak resistively decays. An important experimental figure of merit is the Lundquist number, $\mathrm{Lu}=\tau_{\text {life }} / \tau_{\text {Alf }}$, where $\tau_{\text {Alf }}$ is the Alfven transit time across the spheromak. Since the Alfvén transit time is just the time required for the spheromak to move its length, the
Lundquist number is a dimensionless measure of the distance the spheromak can traverse in its lifetime. In our experiment, $\mathrm{Lu} \cong 10$ so that this condition is marginally met. $\mathrm{A}$ related dimensionless number is the magnetic Reynolds number, $\mathrm{Rm}=\mu_{0} r v / \eta$, where $r$ is a typical spheromak dimension (its radius) and $v$ is its velocity. A large $R m$ is indicative of a high degree of magnetic turbulence. Magnetic turbulence and the associated magnetic tearing is critical for helicity injection experiments. It is because magnetic helicity is conserved even in the presence of turbulent tearing ${ }^{18,19}$ that spheromak injection into a tokamak can be expected to drive current.

As noted earlier, it has recently been suggested by the Los Alamos CTX group that spheromaks might rapidly lose magnetic helicity (and therefore have anomalous lifetimes) due to strong electric fields in the edge. ${ }^{8}$ The CTX group has observed peaking of the current profile (parameterized by $J / B$ ) during the decay phase of the discharge (cooler plasma at the edge causes higher resistivity there).$^{20}$ As the spheromak relaxes back to a flat $J / B$ profile consistent with the force-free Taylor state, ${ }^{19}$ electric fields arise in the edge in order to drive the current. The helicity decay rate is given by:

$$
\frac{d K}{d t}=\dot{K}=-2 \int E \cdot B d V
$$

If we assume that most of the dissipation occurs in the edge where the electric fields are highest then the loss rate can be approximated:

$$
\dot{K} \cong K / \tau_{K} \cong-2 \Phi_{\text {edge }} \int_{\text {edge }} E \cdot d l,
$$

where $\Phi_{\text {edge }}$ is the spheromak magnetic flux either on open field lines or otherwise immersed in the edge volume where there is a significant proportion of neutral gas. The electric field becomes "clamped" at the value given by the Paschen breakdown condition. Note that the resistivity directly attributable to electron-neutral collisions is small compared to the Spitzer resistivity since typically $v_{\mathrm{en}} / v_{\mathrm{ee}}<0.1$ in our experiment (where $v_{\mathrm{en}}$ is the electron-neutral collision frequency).

We can determine the fraction of flux required in the "edge" in order for the edge loss mechanism to have a significant effect on our experiment. The minimum Paschen voltage for breakdown in hydrogen is about $250 \mathrm{~V}$ while the minimum path for edge breakdown is a poloidal circumference, $2 \pi(0.12 \mathrm{~m})=0.75 \mathrm{~m}$. If we assume that only the outer $5 \%$ is the "edge" then the edge flux becomes $5 \times 10^{-5} \mathrm{~Wb}$, where we have used $0.03 \mathrm{~T}$ as the average spheromak magnetic field. We can now estimate an upper bound on $\tau_{\mathrm{K}}$ due to this edge loss mechanism by calculating a lower bound to $\dot{K}$ in Eq. (4). Using a total helicity content ${ }^{10,11,17}$ of about $10^{-7} \mathrm{~Wb}^{2}$, we therefore conservatively estimate $\tau_{K} \leqslant 10 \mu \mathrm{s}$ due to this effect in our experiment (with the assumption of $5 \%$ edge flux ). This is close to our measured $\tau_{\mathrm{K}} \cong \tau_{\mathrm{B}} / 2=5$ $\mu$ so the edge loss mechanism is a plausible candidate for our anomalously short lifetimes. 


\section{B. Zero-dimensional impurity model}

Based on the simple Spitzer lifetime calculation, our anomalously short observed lifetimes could be due to high $Z_{\text {eff }}$ caused by a large impurity content. To investigate this possibility, a 0-D rate equation model for impurity charge state densities was developed similar to that of Barnes et al. ${ }^{7}$ The model includes (i) the loss of neutral hydrogen due to electron impact ionization ( the rate of recombination of protons and electrons to reform neutral hydrogen is negligible at $10 \mathrm{eV}$ ), (ii) the production of electrons due to ionization of hydrogen and impurities, and (iii) loss of electrons due to an empirically determined finite confinement. Ionization, recombination and finite confinement of impurities are also included. The equations also include simple, ad hoc impurity source terms modeling the production of neutral impurities during the formation period of the spheromak when electrode ablation is most severe. The strength of these source terms determines the relative impurity densities and the total impurity percentage for a given program run. The hydrogen density is determined by charge neutrality:

$$
n_{H}=n_{e}-\sum_{j, q} Z_{q} n_{j q}
$$

$Z_{\text {eff }}$ is also calculated at each time step:

$$
Z_{\text {eff }}=\frac{n_{H}+\sum_{j, q} Z_{q}^{2} n_{j q}}{n_{e}} .
$$

The initial conditions for the model are a neutral hydrogen density in accord with a single gas puff (there is no time dependent source of hydrogen), a small seed electron density, and no impurities. The rate coefficients ${ }^{16}$ are calculated assuming a constant, time independent electron temperature.

Carbon lines (CII, CIII) were the brightest impurity lines observed in our cxperiment and no $C V$ lines were observed. Figure 1 shows a program run in which carbon (the only impurity included here) peaks at $25 \%$ of the density. The qualitative temporal behavior of the impurity densities resemble the experimentally measured time evolution of impurity line emission (see, for example, Fig. 5). This run considered only carbon charge states up to and including $C I V$. The electron temperature is assumed to be $10 \mathrm{eV}$. Even with these liberal estimates of $T_{e}$ and the impurity level, we find that $Z_{\text {eff }}$ never rises above 1.3. Similar results are obtained with various fractions of oxygen, aluminum, chromium, iron, nickel, and copper (the other observed impurity lines). Thus, this model excludes the possibility that even a substantial impurity fraction will increase $Z_{\text {eff }}$ to the values of 5-10 needed to account for our observed anomolously short lifetimes. Our anomalous lifetimes must therefore be due to some other mechanism.

\section{CALTECH SPHEROMAK INJECTION EXPERIMENT}

The Caltcch Spheromak Injection Experiment involves the formation and injection of small $\left(r_{0}=4 \mathrm{~cm}\right)$, dense $\left(n_{e}=10^{15} \mathrm{~cm}^{-3}\right)$, high current $\left(I_{\text {gun }}<150 \mathrm{kA}\right)$ spheromaks into the ENCORE tokamak $(r=12 \mathrm{~cm}, R=38 \mathrm{~cm}$,
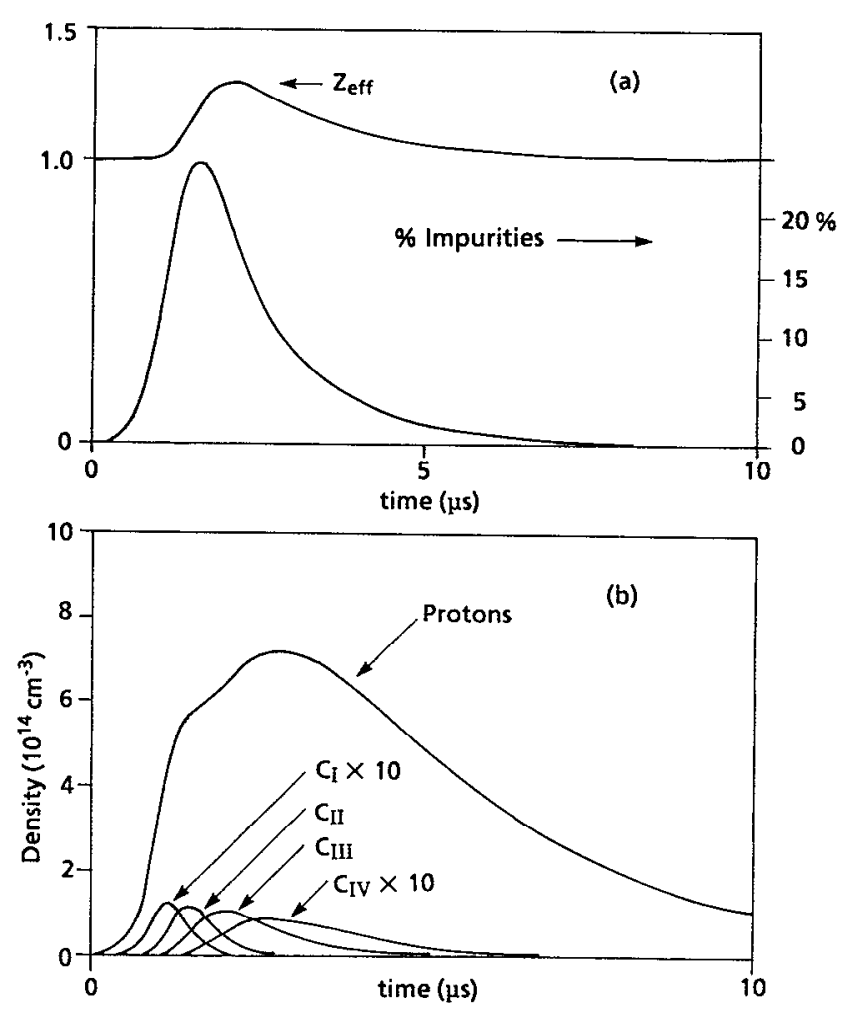

FIG. 1. Plot of result of 0-D, time dependent impurity calculation (carbon only), $T_{c}=10 \mathrm{eV}$, (a) $Z_{\mathrm{efr}}$ (left) and fraction of impurities (right), (b) density of the various ionic components of the plasma, total density $10^{15}$ $\mathrm{cm}^{3}$.

$I_{p}=4 \mathrm{kA}, B_{0}=700 \mathrm{G}$ ) (see Fig. 2). The base pressure is in the low $10^{-6}$ Torr range and titanium gettering reduces pressure to the low $10^{-7}$ range. The injected spheromak deposits density and magnetic helicity into the tokamak discharge. The spheromak is formed by a coaxial, magnetized plasma gun similar to those originally used by Alfvenn. ${ }^{21}$ The inner electrode of the gun is constructed of cold rolled steel or Monel (copper/nickel alloy) $2.5 \mathrm{~cm}$ in diameter. The outer electrode is a tube of stainless-steel $5 \mathrm{~cm}$ in diameter. Hydrogen gas ( 0.1 Torr) is puffed into the annular region between electrodes and a $10 \mathrm{kV}, 6 \mathrm{~kJ}$ capacitor bank initiates the discharge. A radial current sheet forms $(100 \mathrm{kA})$ and accelerates down the gun distending magnetic flux $(0.3$ $\mathrm{mWb}$ ) applied at the end of the gun (often referred to as "stuffing flux") and ultimately forming a spheromak.

Impurities entrained in the spheromak discharge play an adverse role in all aspects of our injection experiments. First, from the standpoint of tokamak refueling, impurities are a deleterious addition to the tokamak discharge. For this reason, it is advantageous to reduce spheromak impurities even if they do not appreciably affect spheromak lifetime. Second, to the extent that impurities reduce the helicity decay time of the spheromak ( $\tau_{\mathrm{K}} \cong \tau_{\mathrm{B}^{2}}=\tau_{\mathrm{B}} / 2$ ), impurities can reduce the amount of helicity deposited into the tokamak discharge. In other words, impurities can reduce the Lundquist number, $\mathrm{Lu}$, so that the spheromak magnetic 
fields decay excessively before reaching the tokamak. Final$l y$, if the spheromak decays substantially before relaxing to its lowest energy state then it is difficult to fit experimental data to a simple (nondynamic) model. ${ }^{17}$

\section{ELECTRODE COATINGS}

Materials used for coating the center electrode of the spheromak gun were chosen for a number of characteristics (see Table I and Refs. 22-24). First, the material should be refractory (high melting point) to prevent melting and vaporization during the high current discharge. Second, the material should have a low sputtering coefficient (where $C_{\text {sput }}$ is the number of electrode atoms evolved per incident plasma ion). The sputtering coefficients presented in Table I represent the number of electrode metal atoms liberated upon impact of a $600-\mathrm{V}$ argon ion. The coefficient for tungsten is a factor of two lower than for other materials in the table so that tungsten is an excellent coating from the standpoint of sputtering.

Secondary emission plays a role in the avalanche breakdown of gas between coaxial electrodes (see appendix B). Ion bombardment of the cathode (the inner electrode in our case) generates secondary electrons which sustain the discharge. Tungsten has about a factor of two higher secondary emission coefficient than other materials for which we have data, so again tungsten has desirable coating characteristics.

Because the discharge has a relatively fast rise time $(<10 \mu$ s, see appendix A), the gun current flows in a skin layer. The skin depth in the cold rolled steel substrate $\delta_{\text {res }}=\sqrt{2 \eta / \mu \omega}$, is less than the coating thickness ( $\delta_{\text {coating }}$ ) because $\mu$ is large. However, the skin depth in the coating is large $\left(\delta_{\text {res }}=\cong 0.4 \mathrm{~mm}=0.015^{\prime \prime}>\delta_{\text {coating }}\right.$ for copper $)$, therefore most of the current flows in the coating for each electrode in our experiment. So, for a skin depth large compared to the coating thickness, the resistive power deposited per unit volume is given by $I^{2} R /$ volume $=I^{2} \eta /\left(2 \pi r \delta_{\text {coating }}\right)^{2}$. Note that if $\delta_{\text {res }}<\delta_{\text {coating }}$ (as it is for nickel), then the power per unit volume for a given current becomes independent of the resistivity $\eta$.
The thermal properties of the coating are governed by the thermal conductivity, $\kappa_{\mathrm{th}}$, and the "thermal mass" of the material (the product of the density and specific heat). A "thermal skin depth" can be defined: $:^{25} \delta_{\mathrm{th}}=2 \sqrt{\kappa_{\mathrm{th}} \Delta t / \rho C_{v}}$ where $\Delta t$ is the time the heat pulse is applied. In each case, the thermal skin depth (for a $10 \mu$ s heat pulse) is comparable to the thickness of the coating. $\delta_{\text {th }}$ varies from 1.2 mil for nickel to $2.7 \mathrm{mil}$ for copper while the electroplated coatings were 1-2 mil thick. Ideally, we would prefer to have the coating thickness large compared to $\delta_{\mathrm{th}}$ so that the coating would act as a thermal reservoir to conduct heat away from the surface. Unfortunately, it is difficult to electroplate a high quality coating thickcr than a few thousandths of an inch and thick plasma-coated surfaces tend to flake off of the substrate (due to internal stresses developed during the coating process).

The steel center electrode of the spheromak gun was electroplated with copper, nickel, chromium, and rhodium (0.001 in. to 0.002 in. thickness) and was plasma coated with tungsten ( $0.006 \mathrm{in}$. thick). After plating, the electrodes were thoroughly cleaned (methanol in an ultrasonic cleaner) and baked to remove residual electrochemicals. Typical base pressures with the coated electrodes were $2 \times 10^{-6} \mathrm{~cm}^{-3}$ with residual gas analysis showing predominantly hydrogen and nitrogen. Each coated electrode was tested under identical conditions ( $V_{\text {gun }}=4.5 \mathrm{kV}, I_{\text {gun }}=70$ $\mathrm{kA}$, and $\Phi_{\text {gun }}=0.3 \mathrm{mWb}$ ) and the visible emission spectrum from the spheromak was measured (18 shots are required to measure the entire visible spectrum from 230 to $890 \mathrm{~nm}$ in roughly $40 \mathrm{~nm}$ increments). Typical plasma densities were $10^{15} \mathrm{~cm}^{-3}$ and electron temperatures were $8 \mathrm{eV}$ in the gun discharge.

The visible spectrum $(230-890 \mathrm{~nm})$ in the annular region between inner and outer electrodes is measured with an optical multichannel analyser (OMA) (Princeton Instruments Model 120, with a 0.22 meter SPEX monochrometer, Model 1681). A 1-mm sealed quartz tube is inserted in the middle of the electrode gap with the tip exposed a few $\mathrm{mm}$ at the back of the gun (see Fig. 2). A quartz optical fiber is inserted into the quartz tube such that emission can be viewed axially along the length of the gun. The line of sight is midway between the inner and outer electrodes. Attenuation

TABLE I. Comparison of refractory electrode coating characteristics.

\begin{tabular}{|c|c|c|c|c|c|c|c|c|}
\hline Material & $T_{\text {melt }}$ & $C_{\text {sput }}$ & $\gamma_{i}$ & $\eta$ & hardness & $\rho$ & $\kappa_{\mathrm{th}}$ & $C_{1}$ \\
\hline Steel & $1550^{\circ} \mathrm{C}$ & 1.3 & 0.061 & 10 & 160 & 7.86 & 0.52 & 0.12 \\
\hline Copper & $1084^{\circ} \mathrm{C}$ & 2.3 & 0.050 & 1.7 & 80 & 8.96 & 4.01 & 0.38 \\
\hline Nickel & $1453^{\circ} \mathrm{C}$ & 1.5 & 0.053 & 6.8 & 140 & 8.90 & 0.91 & 0.44 \\
\hline Chromium & $1860^{\circ} \mathrm{C}$ & 1.3 & - & 13 & 220 & 7.19 & 0.94 & 0.45 \\
\hline Rhodium & $1965^{\circ} \mathrm{C}$ & 1.5 & - & 4.6 & 260 & 12.9 & 1.50 & 0.24 \\
\hline Tungsten & $3400^{\circ} \mathrm{C}$ & 0.6 & 0.096 & 5.6 & 440 & 19.3 & 1.74 & 0.13 \\
\hline
\end{tabular}

Notes: Sputtering coefficient is for dc yield from bombardment of $600-\mathrm{V}$ argon ions, secondary emission coefficient $\left(\gamma_{1}\right)$ is from bombardment of "slow" hydrogen ions, electrical resistivity $(\eta)$ is in units of $10^{-8} \Omega \mathrm{m}$, hardness is in units of $\mathrm{kg} / \mathrm{mm}^{2}$, density $(\rho)$ is in units of $\mathrm{g} / \mathrm{cm}^{-3}$, thermal conductivity $\left(\kappa_{\mathrm{th}}\right)$ is in units of WcIn ' $\mathrm{K}$ ' at room temperature and specific heat $\left(C_{t .}\right)$ is in units of $J_{g}{ }^{-1} K^{-1}$.

References: CRC Handbook of Applied Engineering Science, R. E. Bolz and G. L. Tuve, Eds. (1970), Metals Reference Book, C. J. Smithells, Ed. (1967) and Glow Discharge Processes, B. Chapman (1980). 


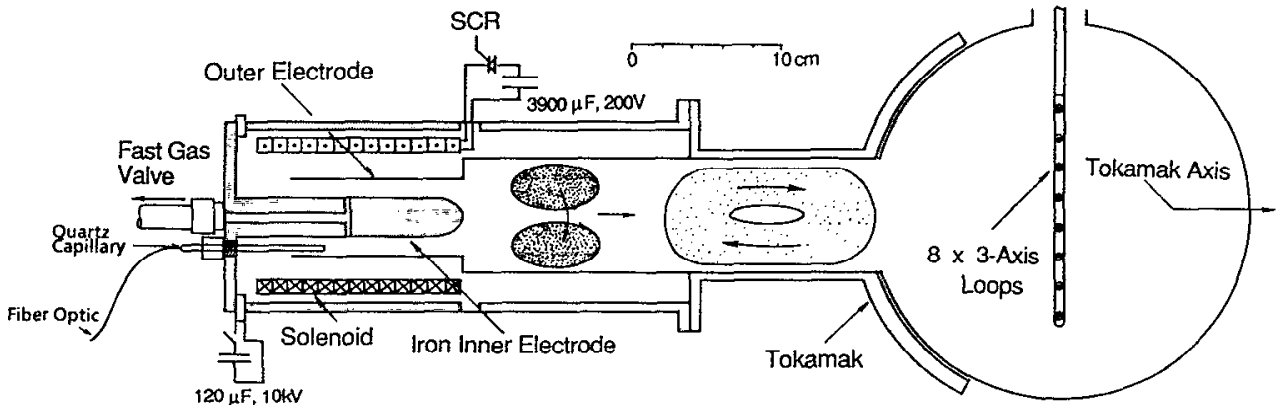

FIG. 2. Schematic of experimental setup showing location of quartz capillary used for optical measurements. along the length of the optical fiber and OMA dynamic range limit our measurements to $230 \mathrm{~nm}$ at the short wavelengths and $890 \mathrm{~nm}$ at the longer. The OMA was gated (10 $\mu \mathrm{s})$ so that light from the gun breech was monitored during only the first half-cycle of the discharge.

The principal result of this sequence of tests is that the spheromak parameters and line emission were largely the same for each electrode (see Table II). There were no systematic differences in spheromak density, temperature, or lifetimes with electrode coating. However, the helicity injection effect ${ }^{10}$ was only observed with the tungsten and chrome electrodes. Small variations in the helicity decay time have a large effect on the amount of helicity that is ultimately deposited into the tokamak since the helicity decay time appears in an exponent $\left(K_{\mathrm{sph}}=K_{0} e^{-t / r \mathrm{~K}}\right)$. Evidently, $\tau_{\mathrm{K}}$ in the case of the "dirty" electrodes was just small enough so that $K_{\text {sph }}$ decayed below our threshold of measurement.

Before the present clean up program was undertaken, we measured lifetimes as low as $15 \mu \mathrm{s}$ and never as long as 25 $\mu \mathrm{s}$, we now routinely measure lifetimes up to $25 \mu \mathrm{s}$ and seldom as short as $15 \mu \mathrm{s}$. This improvement is not due to a specific electrode coating but due to a combination of the use of Macor ceramic insulators, titanium gettering and, most important, effective discharge cleaning from firing hundreds of shots. The best results have been with an uncoated Monel electrode with a Macor insulator after firing $\cong 200$ shots (we observe similar results with a tungsten coated electrode with Macor insulators). Other researchers have made the obser-

TABLE II. Summary of refractory electrode coating results.

\begin{tabular}{lclll}
\hline \hline Material & $\begin{array}{c}\text { Thickness } \\
\text { in. }\end{array}$ & \multicolumn{1}{c}{ Lines suppressed } & Sputtering Helicity \\
\hline Steel & - & - & yes & no \\
Copper & 0.001 & $576.3(\mathrm{FeI})$ & yes & no \\
Nickel & 0.002 & - & yes & no \\
Chromium & 0.002 & $426.7(\mathrm{CII}), 504.1(\mathrm{FeI})$, & no & yes \\
& & $505.1(\mathrm{FeI}), 576.3(\mathrm{FeI})$, & & \\
& & $634.8(\mathrm{FeI}), 637.0(\mathrm{NiI})$ & & no \\
Rhodium & 0.001 & $426.7(\mathrm{CII})$ & yes & yes \\
Tungsten & 0.006 & $426.7(\mathrm{CII}), 504.1(\mathrm{FeI})$, & no & \\
& & $505.1(\mathrm{FeI}), 576.3(\mathrm{FeI})$, & & \\
& & $634.8(\mathrm{Fel}), 637.0(\mathrm{NiI})$ & & \\
\hline \hline
\end{tabular}

vation that firing many shots is the best technique for cleaning spheromak guns. ${ }^{8.26}$ It is therefore likely that the discharge cleaning removes some low- $Z$ impurities (carbon and oxygen) from our electrode surface.

In Fig. 3 we present spectra from six electrodes (tungsten, rhodium, chromium, nickel, copper, and bare steel) and two typical wavelength ranges (487-533 and 606-652 $\mathrm{nm}$ ). The main point is that the spectra are remarkably similar from $230-890 \mathrm{~nm}$. There are several hundred identifiablc lines common to each electrode coating. Next, it is interesting that a few lines are suppressed in the case of the tungsten and chromium coated electrodes (FeI and NiI). The suppressed lines that have been identified are summarized in Table II. Also noted in Table II is an indication whether sputtering onto the quartz capillary was observed and whether the helicity injection effect ${ }^{10}$ was observed. We should note that the limited resolution of the OMA (only a few angstroms) made absolute identification of lines diffcult. The identification of a given line is the most likely candidate of several annotated lines.

In addition to monitoring visible light emission in the breech of the gun, we also scanned the visible spectrum of emission from the tokamak discharge (1/4 of the tokamak circumference from the injection point) with and without spheromak injection. There was much less line emission from the tokamak plasma than from the gun breech and there were only $\sim 10$ additional lines observed upon spheromak injection. In Fig. 4, we present typical data from the wavelength range $527-572 \mathrm{~nm}$. Note first that there are many fewer lines than in the scans presented in Fig. 3. Second, note that two new lines appear as a result of spheromak injection ( $\mathrm{NiI}$ and $\mathrm{FeI}$ ). The fact that we see fewer lines in the tokamak could be due to inefficient transport of high- $Z$ impurities from the ablated electrodes. Other researchers have noted that heavy impurities (mostly FeI in our case) are not efficiently accelerated by the current sheath in the gun. ${ }^{27}$

We noticed that after 30-40 shots, the quartz optics, particularly the $1-\mathrm{mm}$ quartz capillary used to view the gun breech, became coated with metal. The coatings on the quartz capillaries were analyzed using energy dispersive $x$ ray analysis. For the most part, material from each electrode was detected on the capillary used for the experiment. We concluded that some (perhaps most) of the sputtering is from the inner electrode but at least some material is being ablated from the outer electrode. All of the electrodes sput- 
(a)
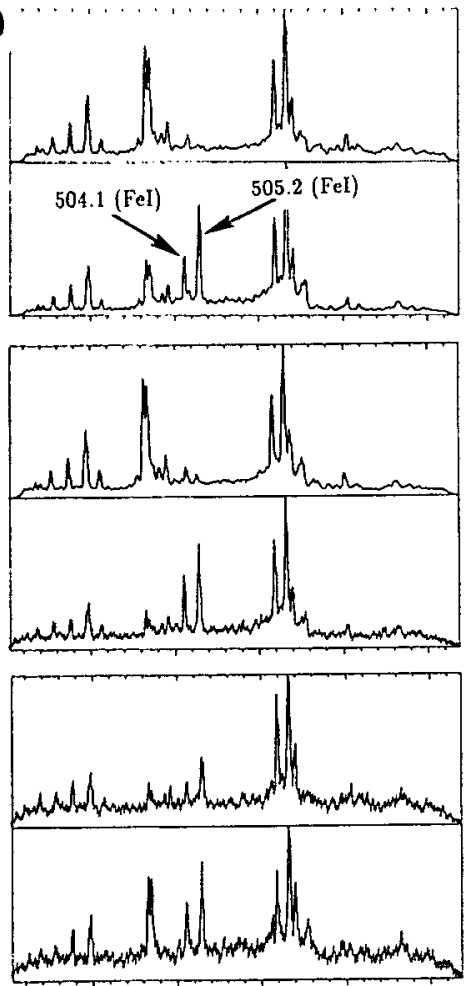

$487 \mathrm{~nm}$
TUNGSTEN

RHODIUM

NICKEL

STEEL

TUNGSTEN

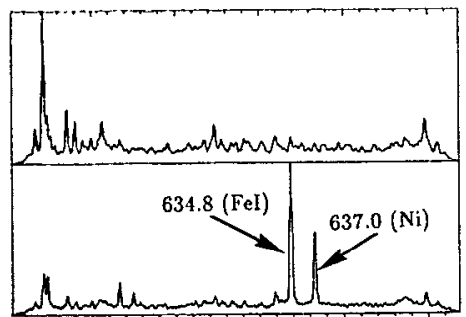

RHODIUM

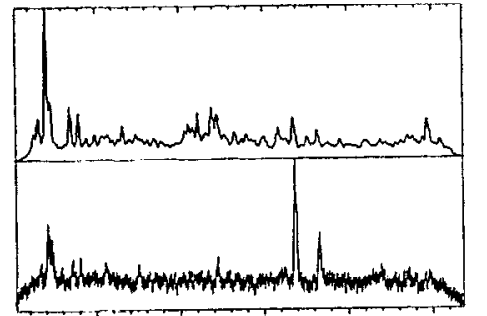

CHROMIUM

NICKEL

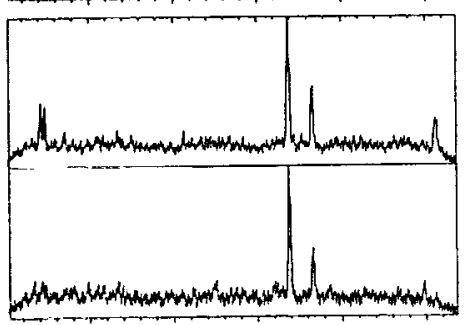

COPPER

STEEL

$606 \mathrm{~nm}$

$652 \mathrm{~nm}$
CHROMIUM

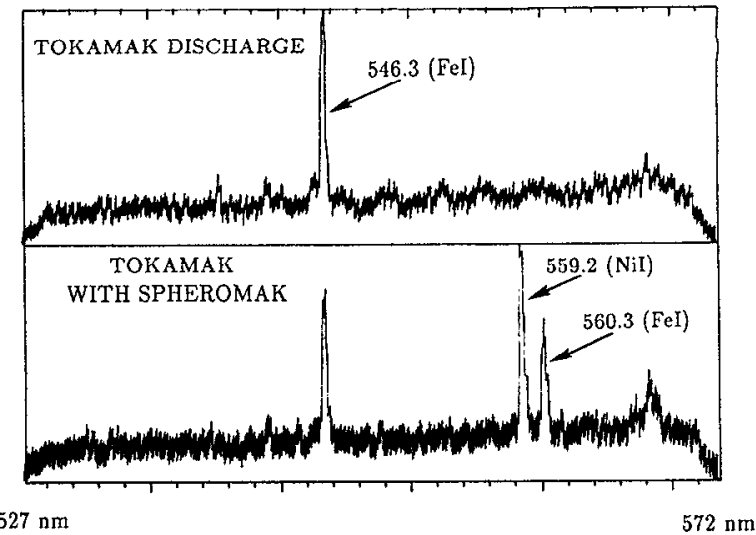

FIG. 4. Typical emission spectrum in the tokamak discharge (top) and tokamak discharge with sphcromak injection (bottom) for the tungsten coated electrode, range from 527 to $572 \mathrm{~nm}$.

tered some material onto the quartz capillary with the notable exceptions of the chromium and tungsten coated electrodes.

In Fig. 5, we present the time history of a resonant CIII line $(229.7 \mathrm{~nm})$ from different electrode/insulator systems. The CIII line is among the brightest impurity lines in our

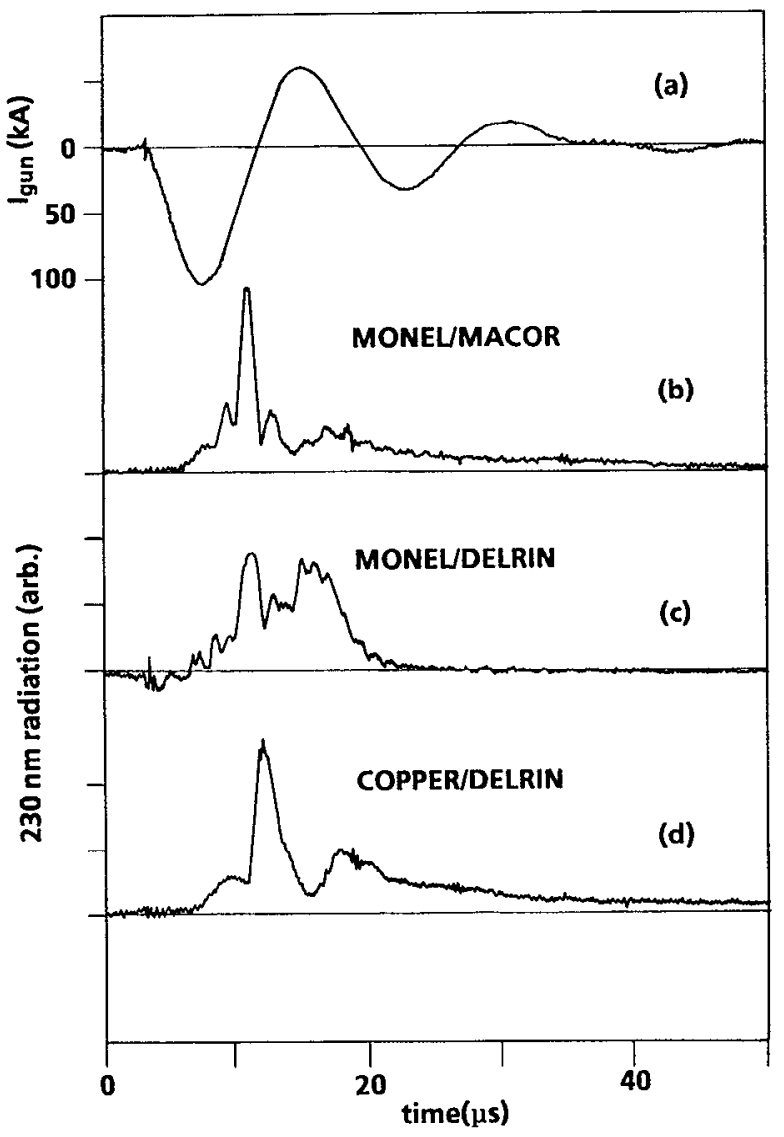

FIG. 3. Typical spectral comparison for each electrode coating, emission from the gun breech, (a) the range from 487 to $533 \mathrm{~nm}$ showing the suppression of some FeI lines in the tungsten and chromium electrodes, (b) the range from 606 to $652 \mathrm{~nm}$, showing supression of $\mathrm{Fel}$ and $\mathrm{NiI}$ lines in the tungsten and chromium electrodes.
FIG. 5. Time histories of $230 \mathrm{~nm}$ emission (including CIII at $229.7 \mathrm{~nm}$ ) in the tokamak discharge upon spheromak injection for representative electrode/insulator systems, (a) $I_{\text {gun }}$, (b) $230 \mathrm{~nm}$ emission with the Monel/Macor system, (c) Monel/Delrin, (d) copper coated/Delrin. 
discharge and we expect emission from carbon lines to be indicative of ablation from plastic insulators. For this measurement, we view emission through a quartz window and narrow band filter (FWHM of $9 \mathrm{~nm}$, centered at $230 \mathrm{~nm}$ ) with a UV enhanced photodiode (UDT-455UV) sensitive down to $200 \mathrm{~nm}$. The window is located on the tokamak vessel, $1 / 4$ of a toroidal circumference from the spheromak injection point.

Our "cleanest" system is a bare Monel electrode and a Macor insulator [Fig. 5(b) ]. We expect our "dirtiest" system to be the high sputtering coefficient, low melting point copper coated electrode with the Delrin (a machinable plastic) insulator [Fig. 5(d) ]. Note however that the emission traces are remarkably similar in magnitude and shape. In each trace there is an initial burst of CIII emission when the spheromak enters the tokamak chamber followed by another burst during the decay phase as the spheromak plasma expands past the viewing window. We see that the time evolution of this typical line is similar for the various electrodes. The macroscopic parameters were also the same for each of these electrode systems. Contrary to expectations, the plastic insulator did not contribute appreciably to the carbon impurity content of the discharge.

\section{DISCUSSION}

In summary, we have coated the center steel electrode of the coaxial, magnetized plasma gun used in our spheromak injection experiments with various refractory metals (copper, nickel, chromium, rhodium, and tungsten). This was done in an attempt to reduce the level of sputtered high- $Z$ impurities into the spheromak discharge so that magnetic lifetimes might be increased. The chromium and tungsten coated electrodes performed marginally better than the others in that we measured only trace amounts of sputtered chromium on our quartz capillaries and we were able to observe the helicity injection effect with these electrodes. In addition, despite the very similar spectra among electrode materials, a small number of spectral lines were supressed in the chromium and tungsten electrodes and not in the others. However, we found that none of the coatings had a measurable effect on spheromak magnetic lifetime, temperature, or density. In addition, discharge cleaning had only a marginal effect on spheromak lifetime (not enough to account for the factor of 5-10 discrepancy between theory and experiment ). On this experimental evidence coupled with a 0-D calculation that $Z_{\text {eff }}<2$ in our discharge (Sec. II B) we conclude a mechanism unrelated to impurities causes our anomalously short lifetimes.

It is instructive to compare our magnetic lifetime measurements with those of other experimenters. In Fig. 6, we compare our longest magnetic lifetime to those measured in several spheromak experiments (data from these experiments are obtained from Refs. 1,6,9,16,28-31). We compare only with other low temperature experiments $(<50 \mathrm{eV})$ since spheromak lifetime in hot spheromaks is governed by dynamic processes rather than simple Spitzer resistivity (e.g., current driven instabilities ${ }^{32}$ and ballooning ${ }^{33}$ ). The reported experimental spheromak lifetime, $\tau_{\text {life }}$, is plotted as a function of $r^{2} T_{e}^{3 / 2}$ where $r$ is the flux conserver radius.
Since $\tau_{\text {life }} \cong \mu_{0} /\left(\eta \lambda^{2}\right)$ and $\eta \propto T^{-3 / 2}$, the data should be linear when plotted this way. A straight line with $r^{2} T_{e}^{3 / 2}$ scaling and passing through the Caltech measurement is plotted (i.e., if we made our spheromak bigger and hotter we would expect to move along this line) as is the Spitzer prediction with $Z_{\text {eff }}=1$. Note first that spheromak lifetimes in this low-temperature regime indeed have the predicted Spitzer-like scaling but with an anomalously low coefficient. This cannot be explained with a large $Z_{\text {eft }}$ since the temperature is not high enough to make $Z_{\text {eff }}$ greater than about two. It appears from the plot that the $20 \mu$ s lifetime of the Caltech spheromak is not anomalous in the context of results from other experiments and that a mechanism unrelated to $Z_{\text {eff }}$ may be a ubiquitous feature limiting lifetimes of cold spheromaks.

We should point out that contrary to this observation, some experimenters have found spheromak $\tau_{\text {life }}$ invariant with size ${ }^{8}$ and with temperature ${ }^{34}$ under certain conditions. However, these results were obtained in the range $r^{2} T_{c}^{3 / 2}=10$ to $100 \mathrm{~m}^{2} \mathrm{eV}^{3 / 2}$ where other processes keep $\tau_{\text {life }}$ "clamped" near $1 \mathrm{~ms}$. Note that data points in Fig. 6 at high $r^{2} T_{e}^{3 / 2}$ begin to deviate below the empirical fit. Recently, Wysocki et $_{\text {al. }}{ }^{35}$ have shown that careful design of the CTX flux conserver has reduced field errors and reduced edge flux so that C'TX spheromak decay time increases with $T_{e}$.

We suggested earlier (Sec. II A) that the edge helicity decay mechanism ${ }^{8}$ may play a role in determining $\tau_{\text {life }}$ of our spheromak. A non-negligible component of unionized neutral gas is unavoidable in the spheromak formation process. Several hundred mTorr of hydrogen must be deposited in

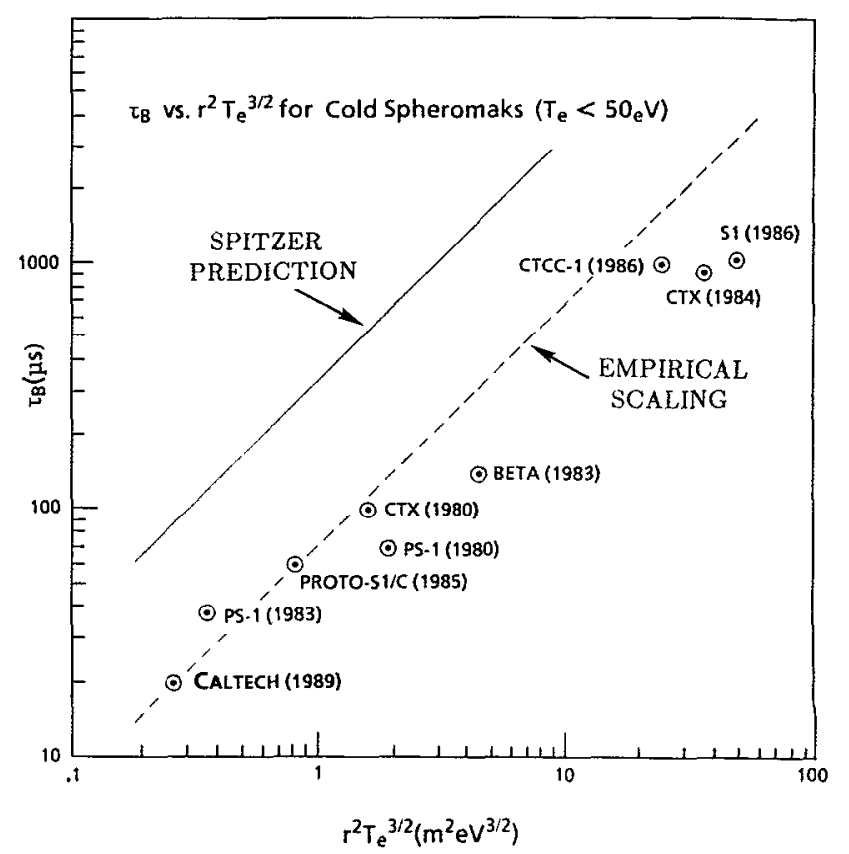

FIG. 6. Plot of $\tau_{\text {life }}$ vs $r^{2} T^{3 / 2}$ for representative small, cold spheromaks. Expected empirical scaling and the Spitzer prediction are also plotted. Note that the measured lifetimes fall below the Spitzer prediction and that data at larger $r^{2} T^{3 / 2}$ fall below the empirical prediction. 
the gun breech (see discussion in Appendix B) some of which is not ionized. In addition, since the spheromak plasma is relatively cool and dense, significant recombination might be expected in the edge. Finally, recent studies have shown ${ }^{34}$ that a large concentration of neutrals can exist within the spheromak far from the edge due to "multiple generation charge exchange". This effectively increases the thickness of the "edge" (since neutrals are present far from the wall) as well as increasing the energy lost due to charge exchange with the neutrals. Based on the simple estimate presented in Sec. II A, edge helicity dissipation may be an important loss mechanism in our spheromak discharge.

\section{APPENDICES: SPHEROMAK GUN CHARACTERIZATION AND OPERATION}

\section{APPENDIX A: ELECTROMAGNETIC PROPERTIES}

In addition to the spectroscopic measurements described in Sec. V, a number of auxiliary diagnostics have been used to characterize the spheromak gun and equilibrium. Various arrays of magnetic probes have been used to measure the magnetic structure of the spheromaks and to determine the magnetic lifetime. ${ }^{17}$ A typical loop in these arrays is $0.5 \mathrm{~cm}$ in diameter and is electrostatically shielded with a slit copper foil. Both active and passive integrators have been used. Double tipped and single tipped Langmuir probes calibrated with a $3 \mathrm{~mm}$ interferometer are used to monitor plasma density. Calibrated Rogowski loops have been used to monitor the spheromak gun current and precision voltage dividers monitor the voltage that appears between the inner and outer electrodes of the gun.

In Fig. 7, we present typical electromagnetic characteristics of the gun (treating it as simple circuit). We present data of the gun current $\left[I_{g}\right.$, Fig. $\left.7(\mathrm{a})\right]$, the gun voltage (the voltage that develops between the inner and outer electrodes during formation, $\left[V_{g}\right.$, Fig. $\left.7(\mathrm{~b})\right]$, a typical signal from a magnetic pickup loop [ $B_{\mathrm{sph}}$, Fig. $\left.7(\mathrm{c})\right]$, and a typical signal from the saturated double Langmuir probe $\left[n_{e}\right.$, Fig. $\left.7(\mathrm{~d})\right]$. The capacitor voltage was $4.2 \mathrm{kV}$ and the stuffing flux was $0.3 \mathrm{mWb}$ for this sequence. Note that $V_{g}$ is determined by dynamic gun impedance and is usually significantly lower than the capacitor voltage. We can determine the point at which the spheromak breaks away from the gun by the characteristic signatures in the $I_{g}$ and $V_{g}$ traces at about $4 \mu \mathrm{s}$. As the gun plasma distends the stuffing flux, the current path is extended thereby rapidly increasing the gun inductance. Since $V_{g}=(d / d t)\left(L_{\mathrm{gur}} I_{\mathrm{g}}\right)$, there is a sharp increase in $\left|V_{g}\right|$. When the spheromak tears away from the gun, the gun discharge restrikes at the back of the gun, rapidly decreasing the gun inductance. At this point there is a drop in $\left|V_{g}\right|$. We can also measure the spheromak velocity by noting that there is a $7 \mu$ s delay between spheromak formation and detection of spheromak fields and density $30 \mathrm{~cm}$ away; the spheromak velocity is $4 \mathrm{~cm} / \mu$ s in this case. Finally, we are able to ascertain the gun stuffing threshold, ${ }^{36} \lambda_{\text {gun }}=\mu_{0} I_{g} /$ $\Phi_{g}$, by noting that the spheromak breaks away from the gun when $I_{g} \cong 50 \mathrm{kA}$ while $\Phi_{g}=0.3 \mathrm{mWb}$. We find that $\lambda_{\text {gun }}$ $=210 \mathrm{~m}^{-1}$ from this dynamic measurement.
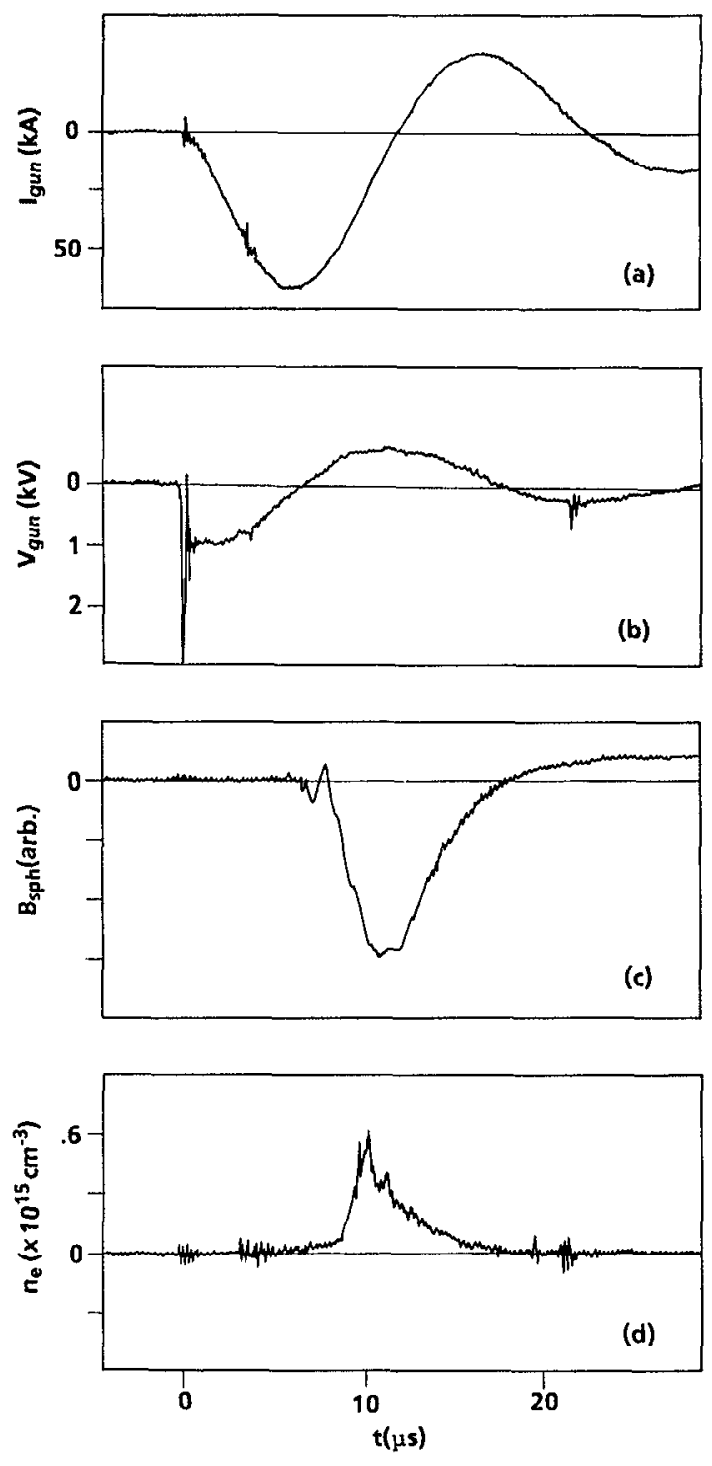

FIG. 7. Typical electromagnetic characteristics of the magnetized plasma gun, (a) $I_{\text {gun }}$, (b) $V_{\text {gun }}$, (c) $B_{\text {spln }}$, and (d) $n_{c}, I_{\text {gun }}$, and $V_{\text {gun }}$ are measured at the gun electrodes, $B_{\mathrm{vph}}$ and $n_{c}$ are measured in the tokamak vessel.

We have performed scans of $I_{g}$ (at fixed $\Phi_{g}$ ) and measured the spheromak magnetic field, density and resistance of the gun plasma with both 2 and 12 external cables. Gun plasma resistance is defined as the ratio of $V_{g}$ at the peak of gun current (to eliminate inductive effects) to the peak $I_{g}$. The fixed stuffing flux for this run was $0.4 \mathrm{mWb}$. The switch from 2 external cables to 12 reduced the total inductance of our power supply and spheromak gun from 660 to $240 \mathrm{nHy}$. This reduced the rise time of the circuit from $\tau_{1 / 4}=14$ to 8.4 $\mu$ s and enabled us to make shorter, more localized spheromak plasmas. We were also able to operate at higher peak current for the same capacitor voltage (since the capacitor charge is switched faster). In Fig. 8(a), note that we begin to see small spheromak fields when $I_{g} \cong 60 \mathrm{kA}$ (below this value the gun is stuffed). From this measurement we find the stuffing threshold, $\lambda_{\text {gun }}=\mu_{0} I_{g} / \Phi_{g} \cong 190 \mathrm{~m}^{-1}$.

Our measured gun threshold, $\lambda_{\text {gun }}=190-210 \mathrm{~m}^{-1}$, is 

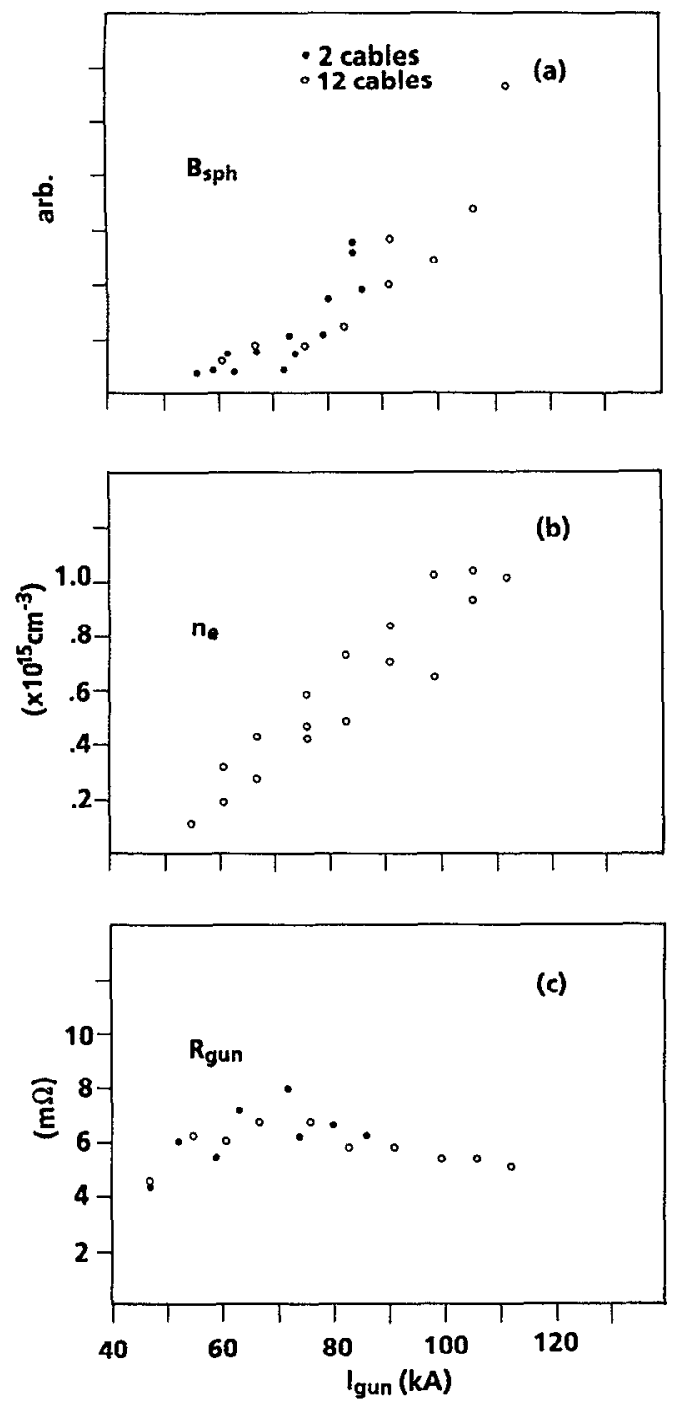

FIG. 8. $I_{\mathrm{gut}}$ scan, (a) $B_{\mathrm{phh}}$, (b) $n_{e}$, and (c) $R_{\mathrm{qun}}$, closed (open) circles are data points obtained with $2(12)$ external cables.

somewhat higher than predicted by recent theories. ${ }^{16,37,38}$ These theories point out that the spheromak gun should be matched to the lowest energy state admitted in the long cylindrical entrance region which has a characteristic energy per unit helicity:

$$
2 \mu_{0} W_{\mathrm{mag}} / K_{\mathrm{sph}}=\mu_{0} I_{\mathrm{g}} / \Phi_{\mathrm{g}}=C_{\mathrm{th}} / r_{\mathrm{ent}}
$$

where $r_{\text {ent }}$ is the radius of the entrance region. The constant, $C_{\mathrm{th}}$, is a number between about 3 and $5\left(C_{\mathrm{th}}=3.11\right.$ if the gun forms a spheromak with $m=1$ symmetry, $C_{\mathrm{th}}=3.83$ for $m=0$ symmetry, $C_{\mathrm{th}} \cong 5.08$ according to a "sharp boundary" model ${ }^{16}$ ). In our case, $3.83 / r_{\text {ent }}=100 \mathrm{~m}^{-1}$ which is a factor of two lower than the measured value of the gun threshold. Barnes et al. ${ }^{38}$ point out that in a coaxial magnetized plasma gun the radial cross section of the flow narrows to form a dynamic "nozzle" which accelerates the flow to the Alfvern speed. The dynamic gun voltage that develops is consistent with (toroidal) flux leaving the gun breech at the Alfven speed. Wc find that our measured threshold is closer to $\pi / \delta_{\text {gap }}$ where $\delta_{\text {gap }}$ is the (narrow) an- nular gap between inner and outer electrode (about $1.3 \mathrm{~cm}$ in our case) where the "nozzle" is formed. Another possible explanation is that driving helicity from the gun to the tokamak through the relatively long and narrow entrance region may be an inefficient process. Fernández et al..$^{39}$ found that the helicity transfer from source to flux conserver was very inefficient through a small cylindrical entrance region (i.e., though the gun threshold was exceeded, the magnetic field devcloped in the flux conscrver was small).

In Fig. 8(b), note that the spheromak density rises rapidly with increasing current. The gas valve pressure was fixed for this run at a plenum pressure of $15 \mathrm{psi}$. We observe only about $10 \%$ increase in spheromak density with a factor of two increase in plenum pressure. We conclude that gas adsorbed on the electrode surfaces could be a possible source of neutrals. Similar observations and conclusions have been made by other experimenters (see Turner et al., ${ }^{16}$ Fig. 24).

In Fig. 8(c), note that the plasma resistance is lower when the gun is stuffed and decreases slightly with increasing current. We can estimate the plasma temperature in the gun breech from the relation $R_{\text {gun }}=\left(\eta / 2 \pi 1_{\text {gun }}\right) \ln \left(r_{2} / r_{1}\right)$ where $l_{\text {gun }}$ is the length of the gun breech and $r_{1}$ and $r_{2}$ are the radii of the inner and outer electrodes respectively. Solving for $T_{e}$, we find that

$$
T_{e}^{3 / 2}=5.2 \times 10^{-5} \frac{Z_{\text {eff }} \ln \lambda \ln \left(r_{2} / r_{1}\right)}{2 \pi l_{\text {gun }} R_{\text {gun }}}
$$

This relationship underestimates the magnitude of $T_{e}$ by a factor of 10 but it points out that a drop in $R_{\text {gun }}$ from 8 to 5 $\mathrm{m} \Omega$ suggests a $25 \%$ increase in $T_{e}$.

\section{APPENDIX B: ROLE OF NEUTRALS}

The operation of the spheromak gun also relies on a large amount of neutral gas delivered to the breech by the action of a fast gas valve. There are several reasons why a high throughput valve is important. First, from the standpoint of tokamak refueling, we would like to generate as high density spheromak as possible. Therefore, we would like to initiate the discharge with the maximum neutral pressure. Second, the Paschen breakdown condition is such that the neutral pressure in the breech of the gun needs to be rather high (a few hundred $m$ Torr) otherwise the breakdown does not occur. Third, if the plasma density is insufficient to sustain the discharge as it progresses, the plasma density will need to be supplemented. If there is insufficient neutral gas available, then electrode material will be ablated (this is the "gas starved" discharge described below). The gas valve necds to open rapidly to prevent neutral gas from propagating ahead of the spheromak before the discharge is initiated.

We have tested several valves and have operated the spheromak gun with two of those tested. In Fig. 9, we plot the response of three different valves as measured by a fast ionization gauge (Schultz-Phelps type $\mathrm{e}^{40}$ of our own design) in a test stand. The gauge consists of two collecting plates 1 $\mathrm{cm}$ square separated by $0.5 \mathrm{~cm}$ and biased at $\pm 45 \mathrm{~V}$. A heated tungsten filament runs midway between the plates. When the gas puff moves across the gauge, the gas is ionized and we measure the ion current to the $-45 \mathrm{~V}$ plate. 
In Fig. 9 (a), we plot the response of a valve designed by Henins of the Los Alamos CTX group. ${ }^{41,42}$ This valve consists of a small plenum $\left(0.6 \mathrm{~cm}^{3}\right.$ in this case $)$ which is pressurized through a small hole with the working gas $\left(\mathrm{H}_{2}\right)$. The plenum is seated at one end of an aluminum rod which is lifted rapidly by magnetic forces from a coaxial solenoid. Firing the solenoid rapidly dumps the contents of the plenum into the system. Note in Fig. 9(a) the abrupt arrival (about $200 \mu$ s rise time) and the narrow width (about 500 $\mu \mathrm{s})$ of the pressure pulse. The peak pressure is about 200 mTorr. This valve gave us the best spheromak parameters.

Initially, we used a small, readily available solenoid valve (Angar Scientific model 008). This valve had a higher inductance solenoid so there was a longer delay before the valve poppet moved. The Angar valve did not have a plenum arrangement and therefore gas flowed as long as the valve was open. Because of the high inductance of the coil the valve remained open for several $\mathrm{ms}$ as is evidenced by the trace in Fig. 9(b). The Angar valve had a sufficiently fast rise time so that we were able to initiate the spheromak discharge on the leading edge of the gas pulse. However, we found that the un-ionized gas trailing the spheromak had a deleterious effect on the spheromak lifetime.

We have tested an automobile fuel injector (GM part No. 17111965) as a possible fast valve for future spheromak gun experiments. The fuel injector has a plenum (about 0.5 $\mathrm{cm}^{3}$ ) which is dumped when the valve is opened. However, unlike the LANL valve, the plenum is recharged through a larger sized hole so that after an initial burst, gas continues to

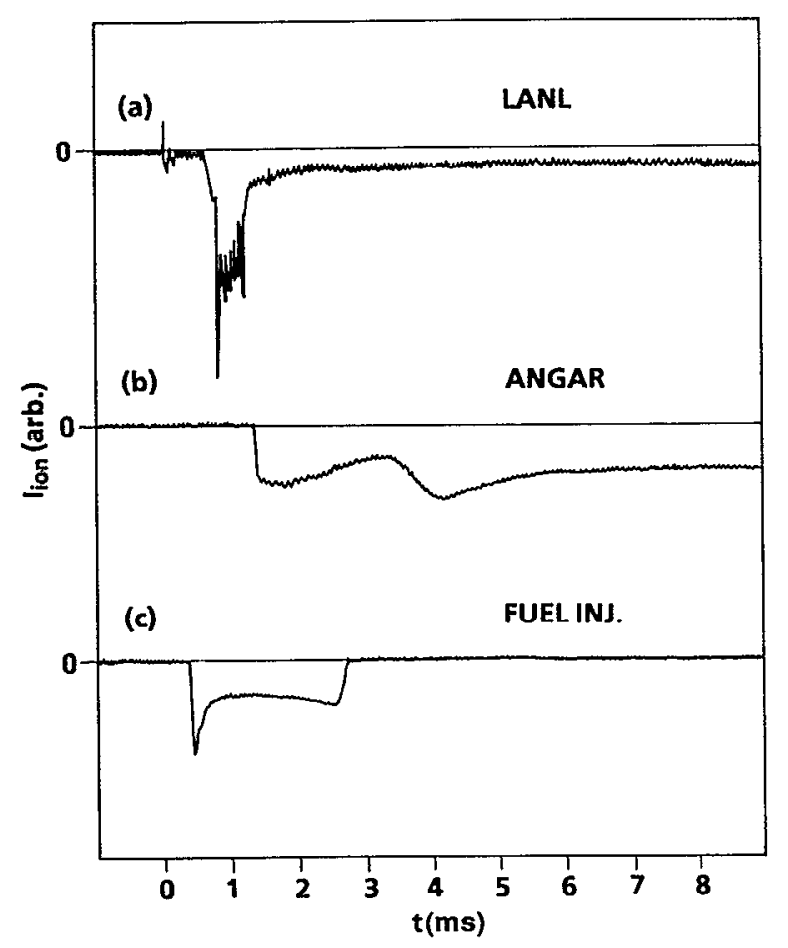

FIG. 9. Plot of valve response measured with fast ion gauge for (a) Los Alamos valve, (b) Angar solenoid valve, and (c) fuel injector. flow as long as the valve is open. The fuel injector has a substantial return spring and relatively low coil inductance so that the valve closes after a short time (about $2 \mathrm{~ms}$ ). In Fig. 9(c), note the initial burst of gas followed by a brief period of flow before the valve closes. The peak pressure in the initial pulse is about $100 \mathrm{mTorr}$. The fuel injector is the primary candidate for a new spheromak accelerator at Caltech. ${ }^{11}$

We have also tested piezoelectric valves with little success. We found that we were unable to initiate a spheromak discharge when a piezoelectric valve was used (even when the valve was "overdriven") and the signal on our fast ionization gauge was small $(<10 \mathrm{mT})$. Evidently, the gas flow through a piezovalve is insufficient for our purposes.

It is interesting to note the role the drift parameter, $J / n_{e}$, plays in these discharges $(J$ is the current density and $n_{e}$ is the electron particle density). $J / n_{e}$ is a useful parameterization of current density in high current discharges. We usually operate with a relatively high value $J / n_{e}=1-4 \times 10^{-14} \mathrm{~A}-m$. First, $J / n_{e}$ is proportional to the drift velocity of the current carrying electrons, so $J / n_{c} T_{e}^{1 / 2}$ is a measure of the ratio of the electron drift to thermal velocity. Generally, if $v_{\text {drift }} / v_{\text {thermal }}$ approaches unity then two-stream instabilities and anomolous resistivity become important. Second, $J / n_{e}$ is a measure of the ratio of ohmic dissipation (heating) to radiation loss $\left(P_{\text {ohmic }}=\eta J^{2}, P_{\text {rad }} \propto n_{e} n_{\text {impurity }}\right)$. Experimentally, it appears that $J / n_{c}$ has an upper bound; the maximum $J / n_{e}=1-5 \times 10^{-14} \mathrm{~A}-\mathrm{m}$ over a wide range of currents and densities in a number of experiments. ${ }^{7,8,43,44}$

Breakdown of neutral gas will occur when primary electron current flowing from the negative cathode is enhanced by multiple ionizations in the traversal to the anode. It is straightforward to show ${ }^{45}$ that the multiplication of primary current between parallel plates is given by:

$$
\frac{I}{I_{0}}=\frac{e^{\alpha d}}{1-\gamma\left(e^{\alpha d}-1\right)},
$$

where $\alpha$ is the electron production per $\mathrm{cm}, \gamma$ is the secondary emission coefficient, and $d$ is the plate separation. Physically, there is the expected exponential growth of the primary current due to multiple ionization but if there is sufficient secondary emission from ion bombardment at the cathode then an "avalanche breakdown" occurs when $\gamma\left(e^{\alpha d}-1\right)=1$. In a coaxial system, $\alpha d$ is replaced by $\int_{r_{1}}^{r_{2}}$ $\alpha d r$ where $r_{1}$ and $r_{2}$ are the inner and outer electrode radii.

Operationally, we have found that running with a negative center electrode affords more reliable breakdown than running with a positive center electrode. This could be due to the higher cathode electric field (due to cylindrical geometry) which may facilitate the generation of primary current or enhance secondary emission at the cathode surface. In any case, negative center electrode coaxial system has been shown to have a lower breakdown voltage than a positive center electrode system. ${ }^{45}$ We also found that we had fewer "flashbacks" (arcs from the high voltage flange back to the gas valve housing) when running with a negative center electrode so this mode of operation was safer than running with a positive center electrode. 
We found that it was important to fire sufficiently late into the gas pulse so not to produce "gas starved" discharges. Material is typically sputtered from the cathode (the center electrode in most cases, ours included) due to high voltage ion bombardment. However, material can also be sputtered from the outer anode in the case of a "gas starved" discharge. This occurs when there is not enough neutral fill gas (hydrogen) to provide the ion flow that sustains the current in the radial current sheet between the inner and outer electrode. A large sheath develops and the material is electrostatically pulled from the anode into the plasma. ${ }^{46}$ In order to prevent the metal electrode surface from becoming a source of ions, it might be useful to make the anode a porous, high surface area material (such as plasma sprayed tungsten or titanium loaded with hydrogen as proposed by Post and Turner ${ }^{25}$ ). In this case, gas adsorbed onto the surface of the anode could sustain a gas starved discharge. In fact, the plot of $n_{e}$ increasing with $I_{g}$ depicted in Fig. 8(b) (with fixed gas puff pressure) is suggestive of just such a process.

\section{ACKNOWLEDGMENTS}

It is a pleasure to acknowledge the technical assistance of M \& T Plating, Frank Cosso, and Larry Begay, as well as useful discussions with Dr. Cris Barnes, Dr. Tom Jarboe, and Dr. Juan Fernández of Los Alamos National Laboratory and Dr. Charles Hartman and Dr. Jim Hammer of Lawrence Livermore National Laboratory. This work was performed under DOE Grant No. DE-FG03-86ER53232.

I T. R. Jarboe, I. Henins, H. W. Hoida, R. K. Linford, J. Marshall, D. A. Platts, and A. R. Sherwood, Phys. Rev. Lett. 45, 1264 (1980).

${ }^{2}$ M. Ono, G. J. Greene, D. S. Darrow, C. Forest, H. K. Park, and T. H. Stix, Phys. Rev. Lett. 59, 2165 (1987).

${ }^{3} \mathrm{~J}$. Marshall, Phys. Fluids 3, 134 (1960)

${ }^{4}$ J. W. Mather, Phys. Fluids 8, 366 (1965).

${ }^{5}$ B. C. Stratton, A. T. Ramsey, F. P. Boody, C. E. Bush, R. J. Fonck, R. J. Groebner, R. A. Hulse, R. K. Richards, and J. Schivell, Nucl. Fusion 27, 1147 ( 1987), and A. T. Ramsey, D. Manos, B. C. Stratton, and M. Ulrickson, Bull. Am. Phys. Soc. 34, 1965 (1989).

${ }^{\circ}$ C. W. Barnes, T. R. Jarboe, I. Henins, A. R. Sherwood, S. O. Knox, R. Gribble, H. W. Hoida, P. L. Klinger, C. G. Lilliequist, R. K. Linford, D. A. Platts, R. L. Spencer, and M. Tuszewski, Nucl. Fusion 24, 267 (1984).

${ }^{7}$ C. W. Barnes, T. R. Jarboe, H. W. Hoida, B. L. Wright, R. A. Hulse, and D. E. Post, Nucl. Fusion 25, 1657 (1985)

${ }^{8}$ J. C. Fernández, C. W. Barnes, T. R. Jarboe, I. Henins, H. W. Hoida, P. L. Klinger, S. O. Knox, G. J. Marklin, and B. L. Wright, Nucl. Fusion 28 , 1555 (1988).

${ }^{9}$ N. Satomi, S. Goto, Y. Honda, Y, Kato, M. Nagata, M. Nishikawa, A. Ozaki, T. Takaishi, T. Uyama, K. Watanabe, T. Sato, and T. Hayashi, International Atomic Energy Agency, Eleventh International Conference on Plasma Physics and Controlled Nuclear Fusion Research, Kyoto, Japan (1986)
${ }^{10}$ M. R. Brown and P. M. Bellan, Phys. Rev. Lett. 64, 2144 (1990).

"M. R. Brown and P. M. Bellan, Phys. Fluids B 2, 1306 (1990).

${ }^{12}$ M. N. Rosenbluth and M. N. Bussac, Nucl. Fusion 19, 489 (1979).

${ }^{13}$ C. W. Hartman and J. H. Hammer, Phys. Rev. Lett. 48, 929 (1982).

${ }^{14}$ P. B. Parks, Phys. Rev. Lett. 61, 1364 (1988).

${ }^{15}$ L. J. Perkins, S. K. Ho, and J. H. Hammer, Nucl. Fusion 28, 1365 (1988).

${ }^{10}$ W. C. Turner, G. C. Goldenbaum, E. H. A. Granneman, J. H. Hammer, C. W. Hartman, D. S. Prono, and J. Taska, Phys. Fluids 26, 1965 (1983).

${ }^{17}$ M. R. Brown, D. M. Cutrer, and P. M. Bellan, Phys. Fluids B (in press).

${ }^{18}$ J. B. Taylor, Phys. Rev. Lett. 33, 1139 (1974).

${ }^{19}$ J. B. Taylor, Rev. Mod. Phys. 58, 741 (1986).

${ }^{20}$ S. O. Knox, C. W. Barnes, G. J. Marklin, T. R. Jarboe, I. Henins, H. W. Hoida, and B. L. Wright, Phys. Rev, Lett. 56, 842 (1986).

${ }^{21}$ H. Alfvén, L. Lindberg, and P. Mitlid, J. Nucl. Energy, Part C: Plasma Phys. 1, 116 (1960).

${ }^{22}$ R. E. Bolz and G. L. Tuve, Eds., CRC Handbook of Applied Engineering Science (CRC, Cleveland, OH, 1970).

${ }^{23}$ C. J. Smithells, Ed., Metals Reference Book (Plenum, New York, 1967).

${ }^{24}$ B. Chapman, Glow Discharge Processes (Wiley, New York, 1980).

${ }^{25}$ R. F. Post and W. C. Turner, Proceedings 4th Compact Torus Symposium, (Los Alamos National Laboratory, 1981), p. 153.

${ }^{26} \mathrm{C}$. W. Barnes, I. Henins, H. W. Hoida, T. R. Jarboe, R. K. Linford, J. Marshall, A. R. Sherwood, and M. Tuszewski, Proceedings 4th Compact Torus Symposium (Los Alamos National Laboratory, 1981), p. 125.

${ }^{27}$ H. W. Hoida, C. W. Barnes, I. Henins, T. R. Jarboe, S. O. Knox, J. Marshall, D. A. Platts, and A. R. Sherwood, Proceedings 5 th Compact Torus Symposium (Mathematical Sciences Northwest, 1982), p. 97.

${ }^{28}$ A. Janos, Phys. Fluids 29, 3312 (1986).

${ }^{29}$ H. Bruhns, C. Chin-Fatt, Y. P. Chong, A. W. DeSilva, G. C. Goldenbaum, H. R. Griem, G. W. Hart, R. A. Hess, J. H. Irby, and R. S. Shaw, Phys. Fluids 26, 1616 (1983).

${ }^{30}$ C. Munson, A. Janos, F. Wysocki, and M. Yamada, Phys. Fluids 28, 1525 (1985).

${ }^{31}$ G. C. Goldenbaum, J. H. Irby, Y. P. Chong, and G. W. Hart, Phys. Rev. Lett. 44, 393 (1980)

${ }^{32}$ Y. Ono, R. A. Ellis, Jr., A. C. Janos, F. M. Levinton, R. M. Mayo, R. W. Motley, Y. Ueda, and M. Yamada, Phys. Rev. Lett. 61, 2847 (1988)

${ }^{33}$ F. J. Wysocki, J. C. Fernández, I. Henins, T. R. Jarboe, and G. J. Marklin, Phys. Rev. Lett. 61, 2457 (1988).

${ }^{34}$ R. M. Mayo, C. K. Choi, F. M. Levinton, A. C. Janos, and M. Yamada, Phys. Fluids B 2, 115 (1990).

${ }^{33}$ F. J. Wysocki, J. C. Fernández, I. Henins, T. R. Jarboe, and G. J. Marklin, Phys. Rev. Lett. 65, 40 (1990).

${ }^{36}$ C. W. Barnes, J. C. Fernández, I. Henins, H. W. Hoida, T. R. Jarboe, S. O. Knox, G. J. Marklin, and K. F. McKenna, Phys. Fluids 29, 3415 (1986).

${ }^{37}$ M. J. Schaffer, Phys. Fluids 30, 160 (1987).

${ }^{38}$ C. W. Barnes, T. R. Jarboe, G. J. Marklin, S. O. Knox, and I. Henins, Phys. Fluids B 2, 1871 (1990).

${ }^{39}$ J. C. Fernández, B. L. Wright, G. J. Marklin, D. A. Platts, and T. R. Jarboe, Phys. Fluids B 1, 1254 (1989).

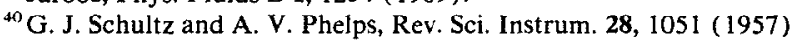

${ }^{41} \mathrm{Kindly}$ provided by I. Henins, LANL.

${ }^{42}$ I. Henins and $\mathbf{J}$. Marshall, Rev. Sci. Instrum. 40, 875 ( 1969).

${ }^{43}$ T. R. Jarboe, Proceedings 7th Compact Torus Symposium (Los Alamos National Laboratory, 1985) p. 34.

${ }^{44}$ H. W. Hoida, C. W. Barnes, I. Henins, T. R. Jarboe, C. J. Buchenauer, and G. Marklin, Proceedings 7th Compact Torus Symposium (Los Alamos National Laboratory, 1985), p. 49.

${ }^{45}$ A. Von Engel, Ionized Gases (Clarendon, Oxford, 1955).

${ }^{4}$ D. A. Platts, T. R. Jarboe, H. W. Hoida, I. Henins, A. R. Sherwood, R. K. Linford, and S. Shimamora, Proceedings 6th Compact Torus Symposium (Princeton Plasma Physies Laboratory, 1984) p. 72. 